Gefördert vom

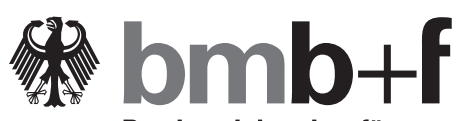

Bundesministerium für

Bildung, Wissenschaft,

Forschung und Technologie

\title{
The Verbmobil Semantic Database
}

\author{
Johannes Heinecke
}

Humboldt-Universität zu Berlin

\section{Karsten Worm}

Universität des Saarlandes 
März 1996

$$
\begin{array}{r}
\text { Johannes Heinecke } \\
\text { Humboldt-Universität zu Berlin } \\
\text { Philosophische Fakultät II } \\
\text { Institut für deutsche Sprache und Linguistik } \\
\text { Computerlinguistik } \\
\text { Jägerstr. 10/11 } \\
10099 \text { Berlin } \\
\text { Tel.: (030) } 20192 \text { - 553 } \\
\text { Fax:(030) } 20196 \text { - } 729 \\
\text { e-mail: heinecke@compling. hu-berlin. de }
\end{array}
$$

Karsten Worm

Universität des Saarlandes

Computerlinguistik

Postfach 151150

66041 Saarbrücken

Tel.: (0681) 302-4503

Fax: (0681) 302-4351

e-mail:worm@coli.uni-sb.de

Die vorliegende Arbeit wurde im Rahmen des Verbundvorhabens Verbmobil vom Bundesministerium für Bildung, Wissenschaft, Forschung und Technologie (BMBF) unter dem Förderkennzeichen 01 IV $101 \mathrm{G}$ gefördert. Die Verantwortung für den Inhalt dieser Arbeit liegt bei den Autoren. 


\section{Contents}

1 Introduction 2

2 The Verbmobil Project 2

3 Motivation and Goals for the Semantic Database 3

4 Design and Implementation of the Database 6

4.1 Semantic Classes . . . . . . . . . . . . . . . . . . . . 6

4.2 The Lexicon Formalism $\mathcal{L E H}^{4} \ldots \ldots \ldots$

4.3 Semantic Classes and their Representation in $\mathcal{L E}^{4} \ldots \ldots$. . . . . . . . 8

4.4 Representation of Lemmata . . . . . . . . . . . . . . . . . . . . . . . 9

5 Application of the Semantic Database $\quad 10$

5.1 Creation of the Semantic Lexicon . . . . . . . . . . . . . . . . . 10

5.2 Table-based Representation . . . . . . . . . . . . . 11

6 Summary 12 


\section{Introduction*}

The distributed development of the modules of a large natural language processing system at different sites makes interface definitions a vital issue. It becomes even more urgent when several modules with the same intended functionality are developed in parallel and should be indistinguishable with respect to their input-output-behaviour.

Another important issue is the acquisition and maintenance of lexical information which should be stored independently of an application to make it (re)usable for different purposes.

This paper describes the design and use of the Verbmobil Semantic Database which we developed in order to deal with these issues in the area of lexical semantics in Verbmobil.

\section{The Verbmobil Project}

The Verbmobil project (Wahlster 1993; Bos et al. 1996) aims at the development of a speech-to-speech machine translation system for face-to-face appointment scheduling dialogues.

The application scenario of Verbmobil is that a speaker of German and a speaker of Japanese try to schedule an appointment. They communicate mostly in English, which they understand better than they speak it. If they they want to say something they cannot express in English, they can have the Verbmobil system translate from both their native languages to English.

The system is being developed by about 30 partners from academia and industry in Germany, the United States and Japan. A first version, the Demonstrator, was completed in early 1994; for autumn 1996 the release of the Research Prototype is scheduled, which marks the end of the first project phase. A second phase is expected to start in 1997.

Verbmobil employs a semantic transfer approach to translation (Dorna and Emele 1996), i. e. an input utterance is syntactically analyzed, a semantic representation of the content is built up, ${ }^{1}$ and this source language semantic representation is mapped to a target language semantic representation by the transfer module. This representation is the input for the target language generation. Additionally, a dialogue processing

* This report slightly modified also appeares in F. Kiefer, G. Kiss, and J. Pajzs (Eds.), Papers in Computational Lexicography: COMPLEX '96, Budapest. A shorter version appeared in D. Gibbon (Ed.), Natural Language Processing and Speech Technology. Results of the 3rd KONVENS Conference, Bielefeld, October 1996. Mouton de Gruyter: Berlin.

${ }^{1}$ Syntactic and semantic analysis proceed in parallel in the Research Prototype, while they were two consequent processing steps in the Demonstrator. 


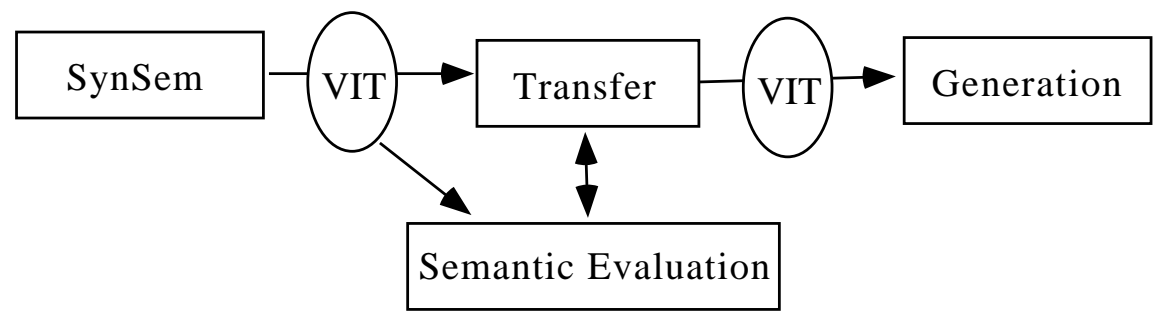

Figure 1: The relevant part of the Verbmobil architecture (simplified)

module and a semantic evaluation module keep track of the discourse and answer disambiguation queries. (The relevant part of the system architecture is shown in figure 1.)

\section{Motivation and Goals for the Semantic Database}

The architecture of Verbmobil makes it necessary for the semantics, transfer, semantic evaluation and generation modules to agree on the format and contents of the semantic representations they exchange. E. g. the developers of the transfer module need to know how the semantics of the different lemmata in the vocabulary is represented in the structures produced by the syntax-semantics module (SynSem for short), i. e. which predicates and structures they have to map to the target language. On the other hand, semantics need to know which readings have to be distinguished by transfer in order to arrive at correct translations.

This need for information becomes even more urgent when, like in Verbmobil, there are several SynSem modules (two for German, one for Japanese), which have to produce compatible output, and the different modules are developed independently and in parallel by several partners at different sites. ${ }^{2}$

As a frame for the exchange of semantic representations a common format, the Verbmobil Interface Term, VIT for short, has been defined (Bos, Egg, and Schiehlen 1996). The VIT is the central data structure used at the interfaces between the language modules of Verbmobil. A VIT is a ten-place term with slots for an utterance identifier, a list of labelled semantic predicates, a pointer to the most prominent predicate, sortal, anaphoric and syntactic information, temporal and aspectual properties, scope relations and prosodic features. Figure 2 shows a VIT for the sentence Wir machen einen Termin aus (We arrange an appointment).

A VIT is an underspecified representation for a set of discourse representation

\footnotetext{
${ }^{2}$ In the following, we concentrate on the Semantic Database for German. The Japanese version follows the same principles.
} 


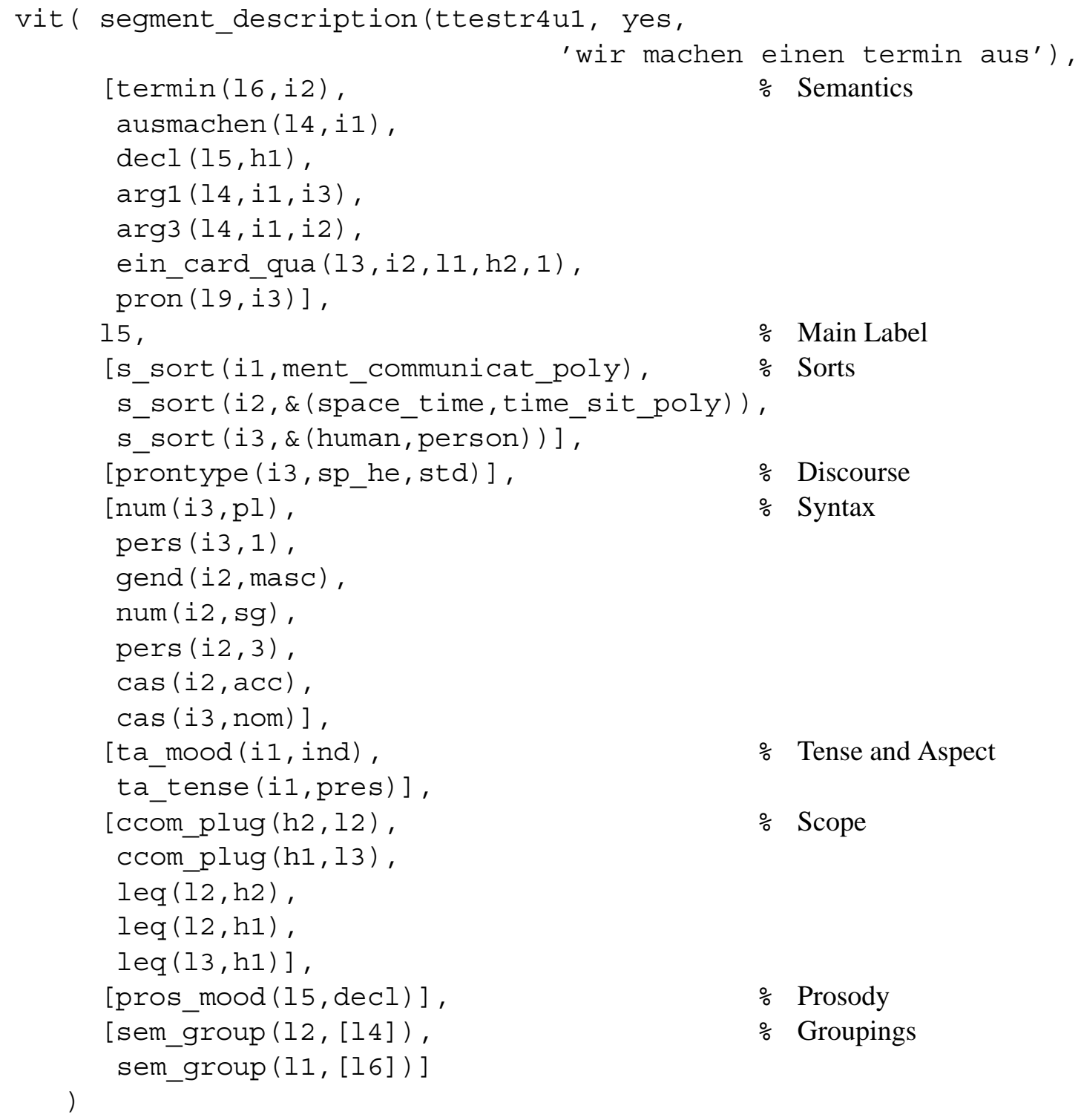

Figure 2: A VIT for Wir machen einen Termin aus ("We arrange an appointment"). 
structures (Kamp and Reyle 1993) in which the scope of operators is not fixed yet. In the example shown in figure 2 both the scope of the declarative sentence mood operator, decl/2, and of the quantifier/indefinite, ein_card_qua/5, are left unspecified. They introduce holes, written as h1 and h2, as their scope, which can be plugged by structures subordinated to them by means of less or equal constraints, written as leq/2. Different ways of plugging the holes result in different readings. In addition to the leq/ 2 constraints determining all possible readings, we supply a default scoping based on syntactic structure in the predicates ccom_plug $/ 2 .^{3}$

All semantic predicates in the VIT are labelled (their first argument is the label). This allows us to group several predicates together (using the semgroup/2 predicate) and form complex substructures which can occur in the scope of operators.

Apart from the purely semantic information mentioned so far, a VIT contains sortal constraints associated with discourse markers, discourse information about anaphoric elements, syntactic agreement and tense information. Since Verbmobil deals with spoken input, we also represent prosodic information in the VIT. ${ }^{4}$

What is needed then in addition to the VIT data structure definition is a definition of the VIT's contents, for each lemma in the vocabulary of the system a definition of the semantic predicates and other types of information, e g. sortal restrictions, it introduces in the slots of the VIT. E. g. for the verb ausmachen in the example above, we need to specify that it introduces a predicate ausmachen $(\mathrm{L} I, I 1)$ together with argument roles $\arg 1(\mathrm{~L} 1, \mathrm{I} 1, \mathrm{I} 2)$ and $\arg 3(\mathrm{~L} 1, \mathrm{I} 1, \mathrm{I} 3)$ in the semantics slot and sort ( $I 1$, ment_communicat_poly) in the sorts slot.

If a source providing this kind of information to the developers of the separate modules is available, the modules which deliver (the two SynSem modules) or process (especially the transfer module) VITs conforming to this definition can be developed in parallel. It would also be desirable to use this information source directly in the construction of the linguistic knowledge bases to guarantee consistency between the output and the specifications.

To meet these goals, we have developed the Verbmobil Semantic Database, which we will describe in the remainder of this paper.

\section{Design and Implementation of the Database}

The semantic database is organized around a set of abstract semantic classes (Bos, Egg, and Schiehlen 1996), which are used to classify the lemmata in the vocabulary. It is

\footnotetext{
${ }^{3}$ For more details on this underspecified approach to semantics, the reader might consult (Bos 1995; Bos et al. 1996).

${ }^{4}$ The VIT in figure 2 has been generated from typed input and thus contains no real prosodic information.
} 


\begin{tabular}{l|l|l} 
Class & PredScheme & Example \\
\hline transitive_verb & $\mathrm{R}(\mathrm{L}, \mathrm{I}), \operatorname{argX}(\mathrm{L}, \mathrm{I}, \mathrm{I} 1), \arg \mathrm{Y}(\mathrm{L}, \mathrm{I}, \mathrm{I} 2)$ & treffen \\
common_noun & $\mathrm{R}(\mathrm{L}, \mathrm{I})$ & Termin \\
det_quant & $\mathrm{R}(\mathrm{L}, \mathrm{I}, \mathrm{H})$ & jeder \\
demonstrative & demonstrative $(\mathrm{L}, \mathrm{I}, \mathrm{L} 1)$ & dieser \\
wh_question & $\operatorname{whq}(\mathrm{L}, \mathrm{I}, \mathrm{H}), \operatorname{tIOC}(\mathrm{L} 2, \mathrm{I} 2, \mathrm{I} 1), \mathrm{time}(\mathrm{L} 1, \mathrm{I} 1)$ & wann
\end{tabular}

Table 1: A few examples of semantic classes

implemented using the lexicon formalism $\mathcal{L E}^{4}$.

\subsection{Semantic Classes}

The semantic classes in use are originally based on a morpho-syntactic classification of the words in the vocabulary of the system which has been refined to account for the semantic properties. This has been decided upon, because words of a certain word-class usually have the same semantic properties. In the example given below, it is shown that transitive verbs all need an instance and two arguments with their semantic/thematic roles.

For each semantic class a representation scheme, called the predscheme, has been defined, which specifies the predicates together with their arity and arguments appearing in a VIT for instances of the class.

As an example consider the class transitive_verb. A transitive verb is represented as $R(L, I), \operatorname{argX}(L, I, I 1), \arg Y(L, I, I 2) .^{5} I$. e., it introduces some relation $R$ and two thematic roles ( $I$ is the event variable, $L$ a label used to refer to the verb's semantic contribution, and II and I2 are the instances filling the roles). The verb's relation and the thematic roles it assigns have to be defined for each verb in the database. Cf. table 1 for further examples of semantic classes together with their predschemes.

\subsection{The Lexicon Formalism $\mathcal{L E}^{4}$}

The semantic database makes use of the lexicon formalism $\mathcal{L E}^{4} 4$ developed in the course of the Verbmobil project (Gebhardi and Heinecke 1995a; Gebhardi 1996).

The Lexicon Formalism $\mathcal{L E}^{4}$ has been used since summer 1994 within Verbmobil's lexicon group. It is based on feature-structures (permitting disjunction and negation) embedded in an inheritance hierarchy of classes.

In $\mathcal{L E}^{4}$ the task of constructing a lexicon is split up into four parts:

\footnotetext{
${ }^{5} \mathrm{X}$ and $\mathrm{Y}$ stand for the values $\{1,2,3\}$, since $\arg 1, \arg 2, \arg 3$ are the thematic roles used in Verbmobil.
} 
1. Modelling the lexicon (i.e. its linguistic classes),

2. data-acquisition (can be done at the same time by different contributors),

3. definition of the application-interface (data can be compiled into every format needed after being processed by the $\mathcal{L E}^{4}$-machine), and

4. efficient storage.

Modelling a lexicon involves defining classes, their appropriate features, and inheritance relations between classes. Examples for defining classes will be given below in section 4.3; appropriateness of features is dealt with in the remainder of this section. For data acquisition, a graphical acquisition tool has been implemented (Heinecke 1996). How the application interface is used in the context of the semantic database will be shown in section 5. Part of the application interface is the $\mathcal{L E}^{4}$-TRAFO which outputs the stored information in any format required. A database system for efficient storage has been developed (Kruschwitz and Gebhardi 1996)

Among other formalism constructs, the possible values of a feature can be specified in two ways. If there is no restriction on the value of a feature, it is assigned the most general value keyword (top):

$$
\text { predname: top . }
$$

Otherwise, the formalism allows to define the appropriateness conditions of a feature, using disjunctions to specify the appropriate values as in the following example (the underlined values are the appropriate ones which can be assigned to the feature sort_of_inst):

$$
\begin{aligned}
& \text { sort_of_inst: ( abstract \ anything \ communicat_sit \ } \\
& \text { communicat_result_poly \person ). }
\end{aligned}
$$

For constructing morphological lexica, inflection or lexical rules can easily be implemented to generate multiple instances of a single entry (Gebhardi and Heinecke 1995b; Heinecke and Gebhardi 1995).

Database entries, called bases, are instances of a class. Consequently, they assign values to the features they inherit from their class which are not yet fully specified by the class definition. For a verb's base, e. g., one has to specify its predicate name, thematic roles, the sort of its instance, etc. 


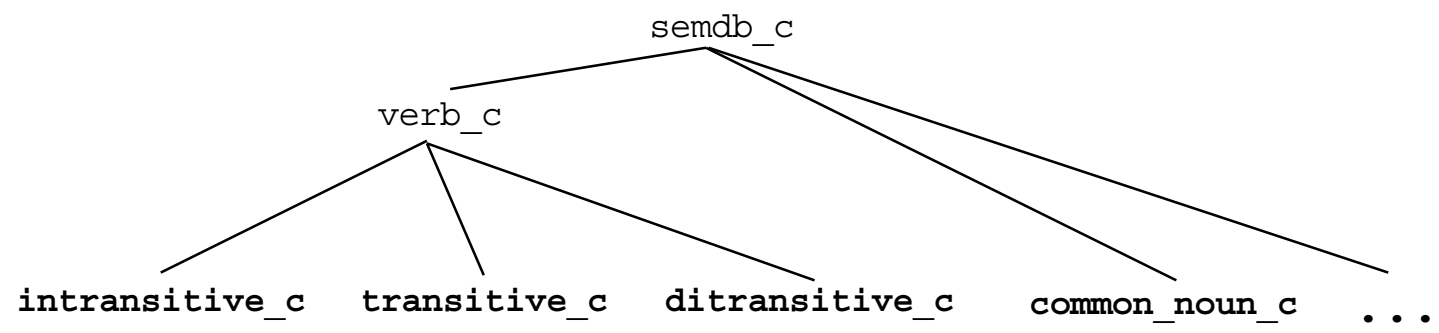

Figure 3: Part of the class hierarchy

\subsection{Semantic Classes and their Representation in $\mathcal{L E}^{4}$}

The abstract semantic classes of section 4.1 have been modelled in the lexicon formalism $\mathcal{L E}^{4}$ along the following lines.

Firstly, a general superclass semdb_c is defined from which all classes inherit features for the lemma, the main predicate's name, the part of speech etc. The individual subclasses corresponding to the abstract semantic classes additionally introduce a specific predscheme for each predicate associated with words of this class and features for sortal information, thematic roles etc.

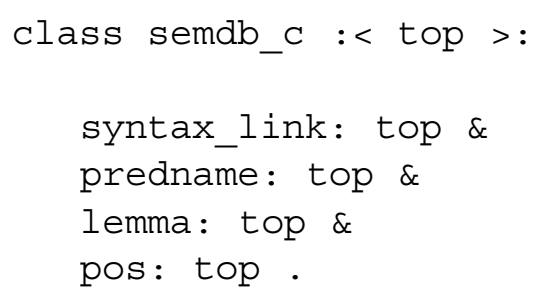
$\%$ - Main class from which all classes inherit.
- Link to syntactic lexicon.
$\div$ - Name of the semantic predicate.
$\%$ - Lemma of the entry.
$\%$ - Part of Speech of the occurrences
$\%$ in the corpora.

While the abstract semantic classes are not hierarchically organized, their modelling in $\mathcal{L E}^{4}$ makes use of a hierarchy to capture generalizations. For instance, we integrate all properties the verb classes have in common and place them in an abstract verb class verb_c from which all verb classes, e. g. transitive_c, inherit, cf. figure 3 (classes corresponding to semantic classes are shown in boldface) and below.

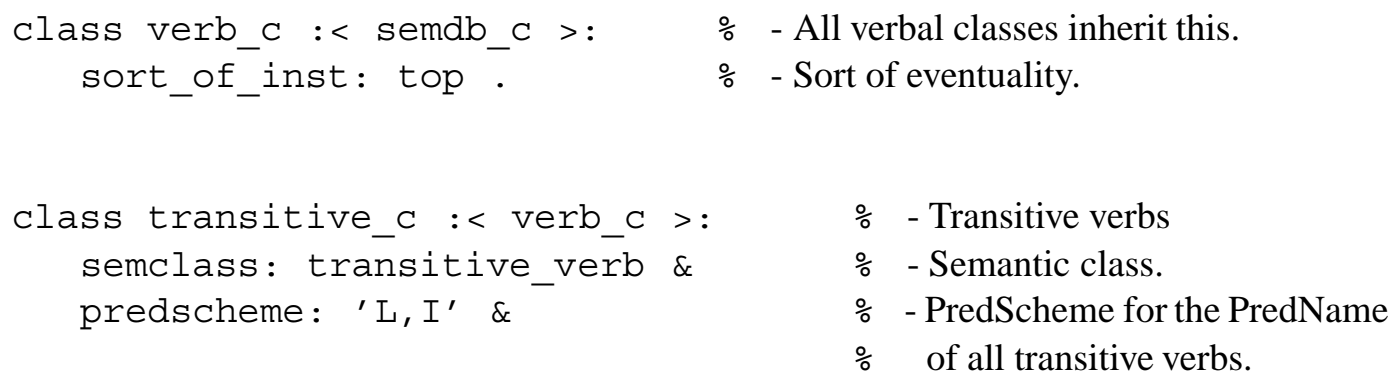




\begin{tabular}{|c|c|c|c|c|c|}
\hline predscheme_a1: & $' \mathrm{~L}, \mathrm{I}, \mathrm{I} 1^{\prime}$ & $\&$ & & $\%$ & - PredScheme for the first \\
\hline predscheme_a2: & $' \mathrm{~L}, \mathrm{I}, \mathrm{I} 2^{\prime}$ & $\&$ & & $\%$ & and the second argument. \\
\hline role_al: (arg1 & $\backslash \arg 2 \backslash$ & $\arg 3)$ & $\&$ & $\%$ & - Thematic roles of the arguments \\
\hline role_a2: & $\backslash \arg 2$ & $\arg 3)$ & - & $\%$ & $\begin{array}{l}\text { of the verb (restricted } \\
\text { to three valid values). }\end{array}$ \\
\hline
\end{tabular}

As a second example, consider the following definition for the $\mathcal{L E}^{4}$ equivalent of the abstract semantic class common_noun:

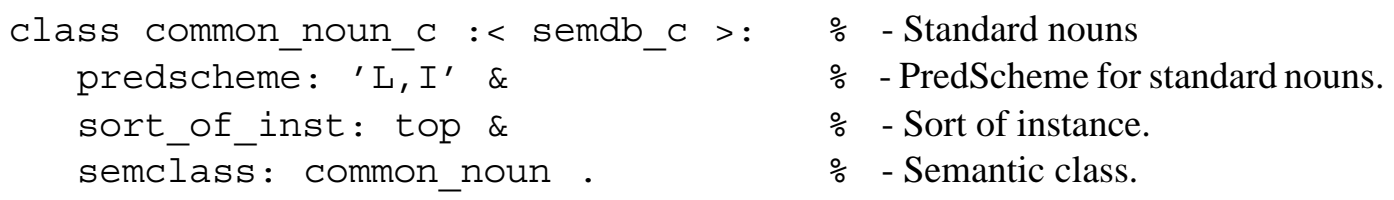

\subsection{Representation of Lemmata}

A base for a lemma consists of its classification together with its idiosyncratic properties in terms of feature values; it inherits the feature values which are specified in the definition of the class. Among the idiosyncratic information we have predicate names, sortal restrictions etc. Thus an entry inherits the predscheme from the class, while the concrete predicate name in the predscheme is defined in the entry itself.

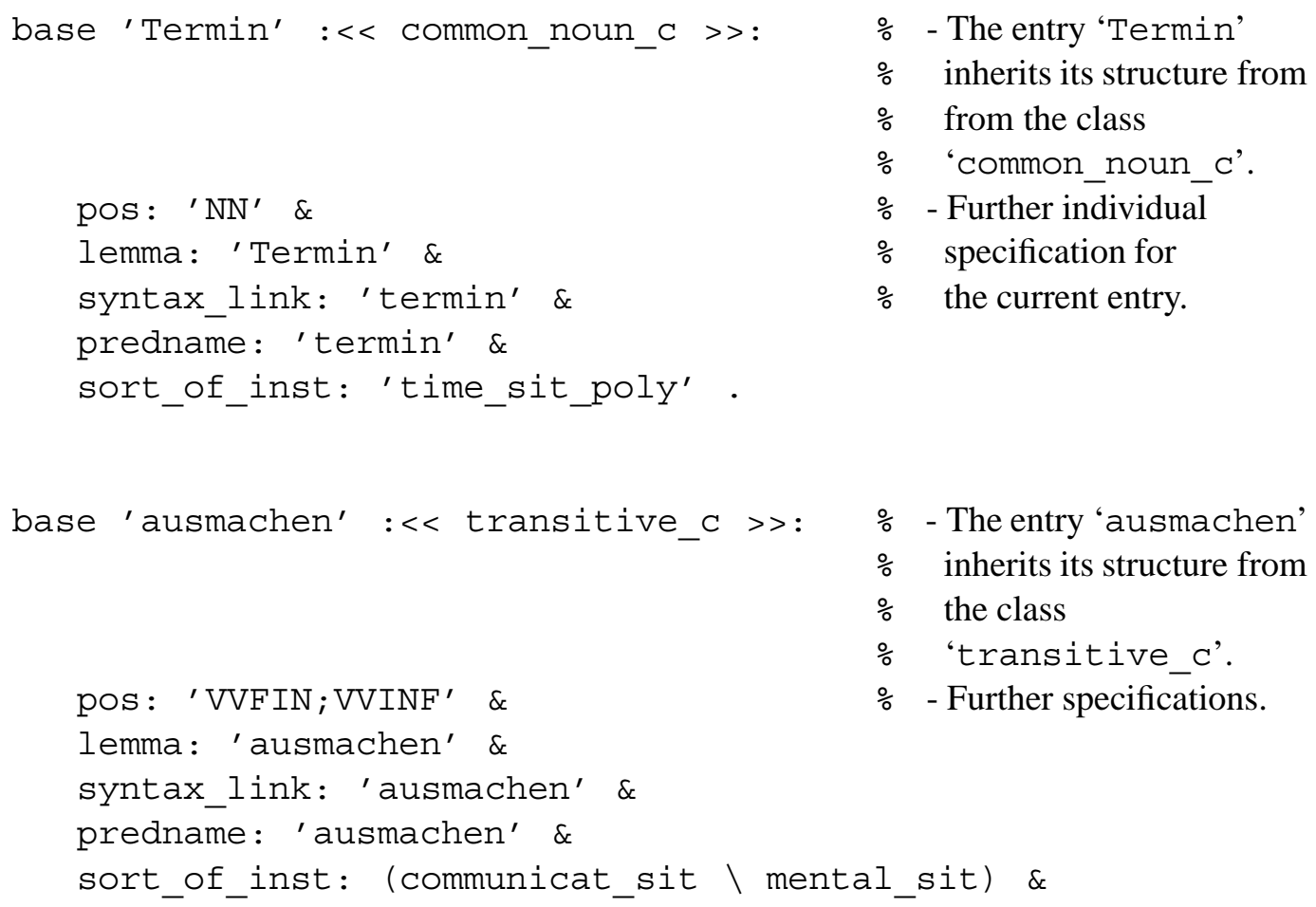




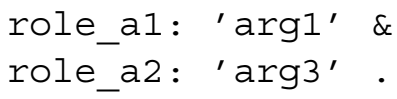

When processing the class definitions and the bases, the $\mathcal{S}^{4} 4$-machine will calculate all instances from the specifications and expand the base accordingly.

\section{Application of the Semantic Database}

The Semantic Database is currently being used for creating the semantic lexica of the syntactic-semantic modules of Verbmobil, for producing a table of lemmata with the predicates and other types of information they introduce in a VIT and for checking the correctness of the generated interface terms automatically; it can also be accessed via the World Wide Web.

A similar procedure is used to generate the semantic lexicon etc. for the Japanese syntactic-semantic module of Verbmobil (Mori 1996).

\subsection{Creation of the Semantic Lexicon}

Consider the compilation of the semantic lexicon from the database for the German SynSem module SynSemS3. ${ }^{6}$ To guarantee consistency between the output of the SynSem module and the specifications in the database, the semantic lexicon is generated out of the semantic database.

After the $\mathcal{L E}^{4}$-machine has processed the entries and expanded them according to the class definitions, the $\mathcal{L E}^{4}-$ TRAFO compiles the $\mathcal{L} \mathcal{E}^{4}$ output into the format required for the semantic lexicon.

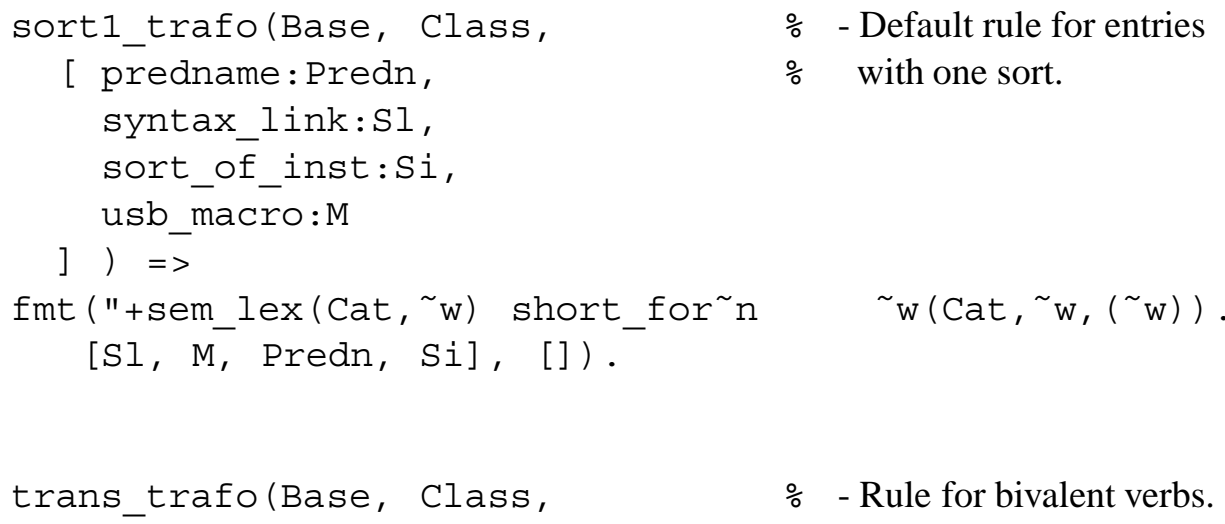

\footnotetext{
${ }^{6}$ SynSemS3 is the syntactic-semantic module developed by Siemens AG (syntax), University of the Saarland and University of Stuttgart (semantics). The other SynSem module developed by IBM Germany makes use of the table output (cf. section 5.2) of the database to create a semantic lexicon.
} 


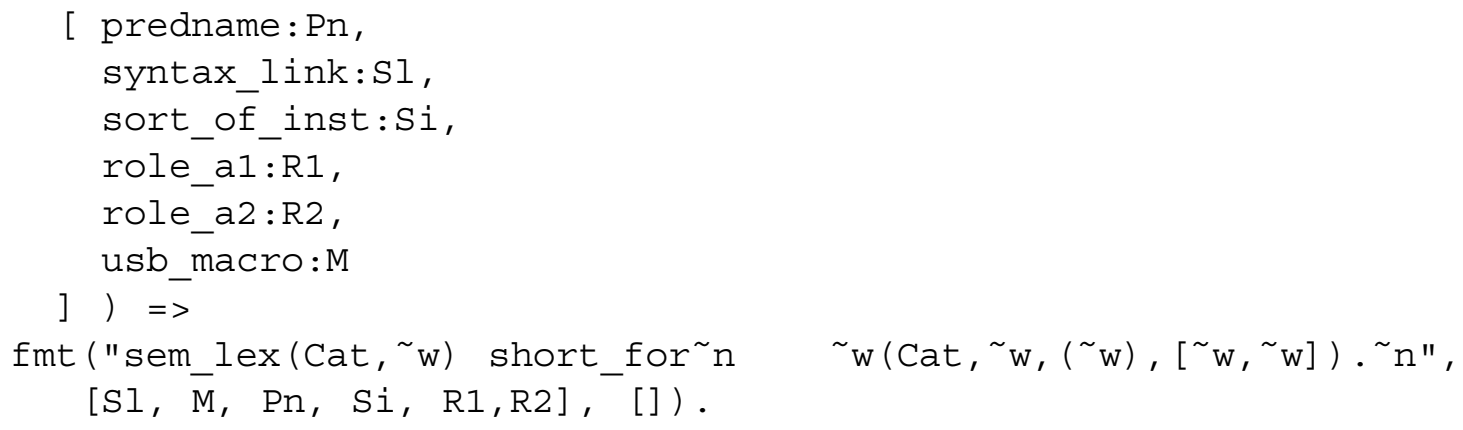

The two examples above appear in the semantic lexicon as:

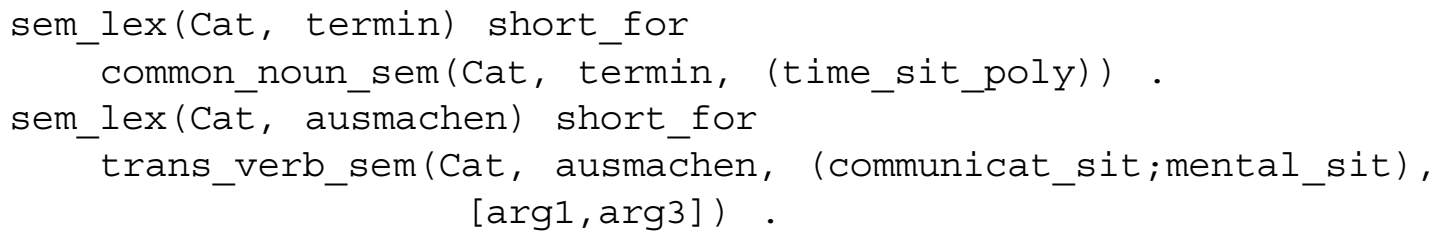

The syntactic lexicon contains calls to the macro sem lex/ 2 which is expanded in the semantic lexicon as shown above. The mapping from syntactic to semantic lexical entries is achieved via the second argument of sem_lex $/ 2$, which originates from the feature syntax_link in the semantic database. ${ }^{7}$

\subsection{Table-based Representation}

Apart from compiling out semantic lexica, we generate a table of lemmata together with their semantic representations and additional information out of the database by using a different set of transformation rules for $\mathcal{L E}^{4}$-TRAFO. This table is used by the transfer developers as a basis for writing transfer rules and as an information source for the automatic correctness check on VIT representations.

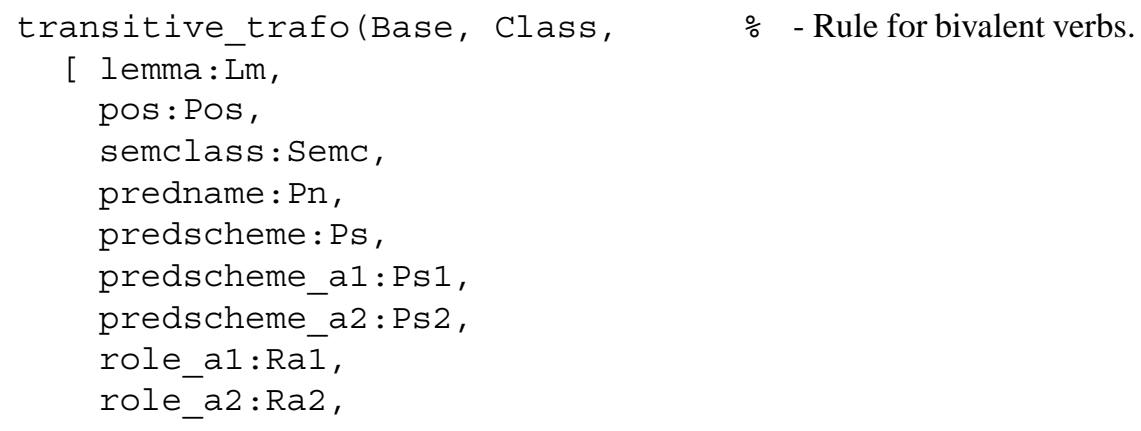

\footnotetext{
${ }^{7}$ The first argument of sem_lex/2 ranges over entry nodes of the feature structures of the lexical entry.
} 


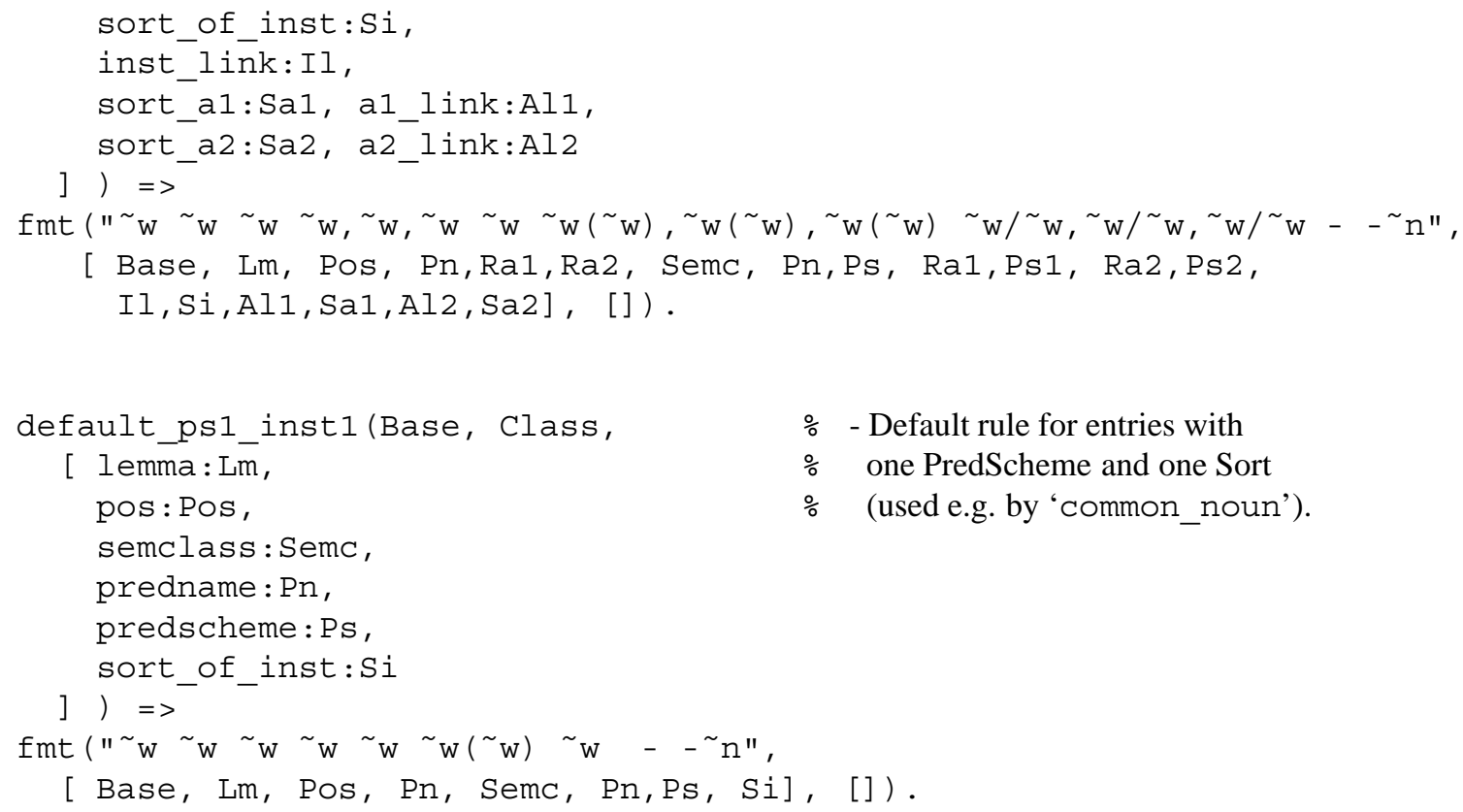

In the table output the two examples above appear as:

Termin Termin NN termin common_noun termin(L,I) I/time_sit_poly - -
ausmachen ausmachen VVFIN; VVINF ausmachen, arg1, arg3 transitive_verb ...
ausmachen $(\mathrm{L}, \mathrm{I}), \arg (\mathrm{L}, \mathrm{I}, \mathrm{I} 1), \arg 3(\mathrm{~L}, \mathrm{I}, \mathrm{I} 2) \mathrm{II} / \mathrm{communicat}$ sit;mental_sit - -

In general the concept of TRAFO is trying to map the output of the $\mathcal{L E H}^{4}$-machine onto the first matching rule in the rule system. Thus only a few class specific rules are necessary, default rules will cover the entries of the majority of the classes to be transformed.

\section{Summary}

We have successfully used the semantic database to deal with about 2000 German and 150 Japanese lemmata for version 1.0 of the Research Prototype in the way described, especially to generate semantic lexica for the German syntax-semantics module SynSemS3, and the Japanese one developed by DFKI Saarbrücken and the University of the Saarland.

The use of the semantic database by both the semantics module and the transfer module guarantees consistency between the representations produced by the semantics module and the expectations of the transfer module, while both can be developed in parallel.

Currently the Semantic Database for the German lemmata can be accessed via WWW at http: / / www. compling.hu-berlin. de/ ${ }^{\sim} \mathrm{vm} /$ semdb. 


\section{References}

Bos, J. (1995). Predicate logic unplugged. In Proceedings of the 10th Amsterdam Colloquium, Amsterdam, The Netherlands, pp. 133-142. ILLC/Department of Philosophy, University of Amsterdam.

Bos, J., M. Egg, and M. Schiehlen (1996). Definition of the Abstract Semantic Classes for the Verbmobil Forschungsprototyp 1.0. Verbmobil-report, Universität des Saarlandes, Computerlinguistik, Saarbrücken.

Bos, J., B. Gambäck, C. Lieske, Y. Mori, M. Pinkal, and K. Worm (1996). Compositional semantics in Verbmobil. In Proc. of the $16^{\text {th }}$ COLING, Copenhagen, Denmark.

Dorna, M. and M. C. Emele (1996). Semantic-based transfer. In Proc. of the $16^{t h}$ COLING, Copenhagen, Denmark.

Gebhardi, G. (1996). $\mathcal{L E}^{4}$ - yet another lexicon formalism. In F. Kiefer, G. Kiss, and J. Pajzs (Eds.), Papers in Computational Lexicography: COMPLEX '96, Budapest.

Gebhardi, G. and J. Heinecke (1995a). Lexikonformalismus LeX4. Verbmobil Technisches Dokument 19, Humboldt-Universität zu Berlin, Computerlinguistik, Berlin.

Gebhardi, G. and J. Heinecke (1995b). Substantivflexion in LeX4. Ein Applikationsbericht. Verbmobil-Memo 62, Humboldt-Universität, Computerlinguistik, Berlin.

Heinecke, J. (1996). Lexikonakquisitionstools für den Lexikonformalismus LeX. Verbmobil Technisches Dokument 42, Humboldt-Universität zu Berlin, Computerlinguistik, Berlin.

Heinecke, J. and G. Gebhardi (1995). Konjugation der Verben im Lexikonformalismus. Verbmobil-Memo 63, Humboldt-Universität, Computerlinguistik, Berlin.

Kamp, H. and U. Reyle (1993). From Discourse to Logic. Dordrecht: Kluwer Academic Publishers.

Kruschwitz, U. and G. Gebhardi (1996). The $\mathcal{L E}^{4}$-database system. In F. Kiefer, G. Kiss, and J. Pajzs (Eds.), Papers in Computational Lexicography: COMPLEX '96, Budapest.

Mori, Y. (1996). Multiple discourse relations on the sentential level in Japanese. In Proc. of the $16^{\text {th }}$ COLING, Copenhagen, Denmark.

Wahlster, W. (1993). Verbmobil: Translation of face-to-face dialogues. In Proceedings of the $3^{\text {rd }}$ European Conference on Speech Communication and Technology, Berlin, Germany, pp. 29-38. 